\title{
DNA-programmed self-assembly of photonic nanoarchitectures
}

\author{
Xiang Lan and Qiangbin Wang \\ Rational design and self-assembly of photonic nanoarchitectures with well-defined structures and geometries allows precisely \\ manipulating light on the nanoscale, which has been the focus of nanophotonics in recent decades. DNA self-assembly is a \\ powerful strategy in constructing desired photonic nanoarchitectures owing to the unique structural features of DNA, such as \\ programmable sequence, predictable structure and precise molecule length $\left(0.34 \mathrm{~nm} \mathrm{bp}^{-1}\right)$. The high addressability of DNA \\ nanoscaffolds enables fine control over the locations of the photonic building blocks and thus the structure and geometry of \\ the assembled photonic nanoarchitectures, which facilitates the quantitative study of the interactions among these photonic \\ building blocks that are precisely organized on the DNA nanoscaffolds. This review summarizes the recent achievements in \\ DNA-programmed self-assembly of photonic nanoarchitectures, where metallic nanocrystals and semiconductor quantum dots \\ act as building blocks and are assembled into homo- and hetero-nanoarchitectures from one to two and three dimensions. \\ NPG Asia Materials (2014) 6, e97; doi:10.1038/am.2014.16; published online 25 April 2014
}

Keywords: DNA self-assembly; gold nanoparticle; gold nanorod; plasmonic nanoarchitectures; quantum dot; surface plasmon resonance

\section{INTRODUCTION}

Over the past years, people have witnessed the development of the flourishing field of nanophotonics, in which tailoring the photonic properties of nanomaterials and manipulating their photonic interactions at nanoscale are the central topics. ${ }^{1-8}$ Most studied photonic nanomaterials are noble metal nanoparticles (MNPs) and semiconductor nanocrystals QDs owing to their distinctive photonic characters. When light interacts with a MNP, collective oscillations of conduction electrons with respect to the positive ion background, known as surface plasmons, can be driven by the incident electromagnetic (EM) field. ${ }^{9}$ With the excitation of plasmon modes, the energy of the incident radiation is significantly harvested and confined at the metal/dielectric interface, generating a strong localized EM field (hot spot) that decays exponentially with the distance away from MNP surface. ${ }^{10}$ The evanescent EM field interactions between the closely spaced MNPs give rise to coupled plasmon modes in a way similar to the atomic orbital hybridization in covalent molecules, which is termed 'plasmon hybridization. ${ }^{11}$ The in-phase and out-of-phase plasmon modes of photonic nanoarchitectures are created through mixing and hybridizing the modes supported by their isolated components. QDs are another type of photonic nanomaterials which are characterized by their particlein-a-box-like energy states. ${ }^{12}$ The valence band is fully occupied by electrons, but the conduction band is rarely occupied. When the energy of the incoming photon is large enough to overcome the band gap, valence electrons can be promoted to the conduction band. The excited states of electrons decay along the inverse path of excitation, and photons with the energy defined by the band gap width are emitted. ${ }^{13}$ Thus, in contrast to MNPs functioning as dielectricconfined plasmon oscillating source, QDs usually work as dipolar quantum emitters with discrete narrow line of light emission spectrum.

To date, a vast assortment of photonic nanomaterials including MNPs and QDs have been successfully achieved with tunable size, shape, composition and structural complexity through wet synthetic chemistry. ${ }^{14-20}$ Whereas, rational assembly of these elementary photonic nanomaterials as building blocks into well-defined photonic nanoarchitectures is the critical prerequisite for the study of the cross-particle interactions and for future optical routing and light switching. ${ }^{21,22} \mathrm{DNA}$, the natural genetic information carrier, is the outstanding material used for directing well-controlled assembly of photonic nanoarchitectures. The Watson-Crick base pairing rule (A-T, C-G) provides strict programmability in assembling the DNA single strands into various precisely-defined templates including DNA double helix, ${ }^{23}$ DNA multi-arm motifs ${ }^{24}$ and DNA tiles, ${ }^{25}$ and so on. Most importantly, the DNA double helix unit in DNA templates has a persistence length of $50 \mathrm{~nm}$ compatible with the dimensions of the photonic building blocks. Therefore, the DNA template can supply significant control over the spatial configuration of the assembled photonic nanoarchitectures. In this review, we highlight the recent 
achievements in the DNA-programmed self-assembly of homo- and/ or hetero-photonic nanoarchitectures comprising MNPs and QDs in one dimension (1D), two dimensions (2D) and three dimensions (3D), and overview their optical properties and potential photonic functionalities. Lastly, we attempt to make our speculation on the challenges and future directions of DNA-programmed self-assembly of nanoarchitectures in the field of nanophotonics.

\section{ONE DIMENSIONAL DNA-PROGRAMMED PHOTONIC NANOARCHITECTURES}

MNPs were first employed for constructing photonic nanoarchitectures through DNA-guided self-assembly. In 1996, for the first time, Alivisatos et al. ${ }^{26}$ obtained gold nanoparticle (AuNP) dimers and trimers through hybridizing complementary DNA monofunctionalized AuNPs. Subsequently, AuNP homodimers, AgNP homodimers and AuNP-AgNP heterodimers were successfully built with high throughput directed by DNA self-assembly. ${ }^{23,27}$ Linear AuNP trimer nanoarchitecture that features interparticle spacing of $<1 \mathrm{~nm}$ can also be obtained through sequential DNA functionalization of each AuNP and subsequent DNA base paring interactions (Figure 1a). ${ }^{28}$ Studies of the ensembles of dimers and a single dimer both showed that the plasmon couplings between particles can produce a dominant bonding plasmon mode with lower resonance energy, thus lead to the red shift of plasmon band in comparison with the isolated particles. When decreasing the interparticle distance by shortening the molecule length of DNA, the plasmon coupling within dimers got strengthened, thus the coupled plasmon band red-shifted. Such distance-dependent plasmonic property enables the DNA-directed plasmonic dimer working as a powerful ruler with resolution down to a few nanometers, which has been experimentally realized. ${ }^{29}$ Besides, dark field scattering measurement of a single plasmonic dimer showed that the plasmon modes of the dimer are also significantly dependent on the polarization of incident EM field. ${ }^{27}$

In the presence of incident EM field, collective oscillations of electron clouds within MNP dimers lead to the accumulation of charge at the nanogap junction thus creating a greatly enhanced EM field in the gap. When an emitter is placed in the nanogap of a MNP dimer, the enhanced EM field in nanoscale volume provides intensive photonic density for excitation of the emitter. The near-field plasmon-emitter coupling will increase the radiative decay rate and decrease the emission life time of the emitter. ${ }^{30}$ Thus, the MNP dimers provide us opportunities to enhance the emission of the emitter located at the junction. An example of this plasmon-enhanced emission was shown by positioning CdSe/ZnS QD in the nanogap of a $80 \mathrm{~nm}$ AuNP dimer that supports strong plasmon resonance, which was realized through surface-bound DNA hybridization between QD and AuNPs. ${ }^{31}$ Single object measurement showed that the emission-
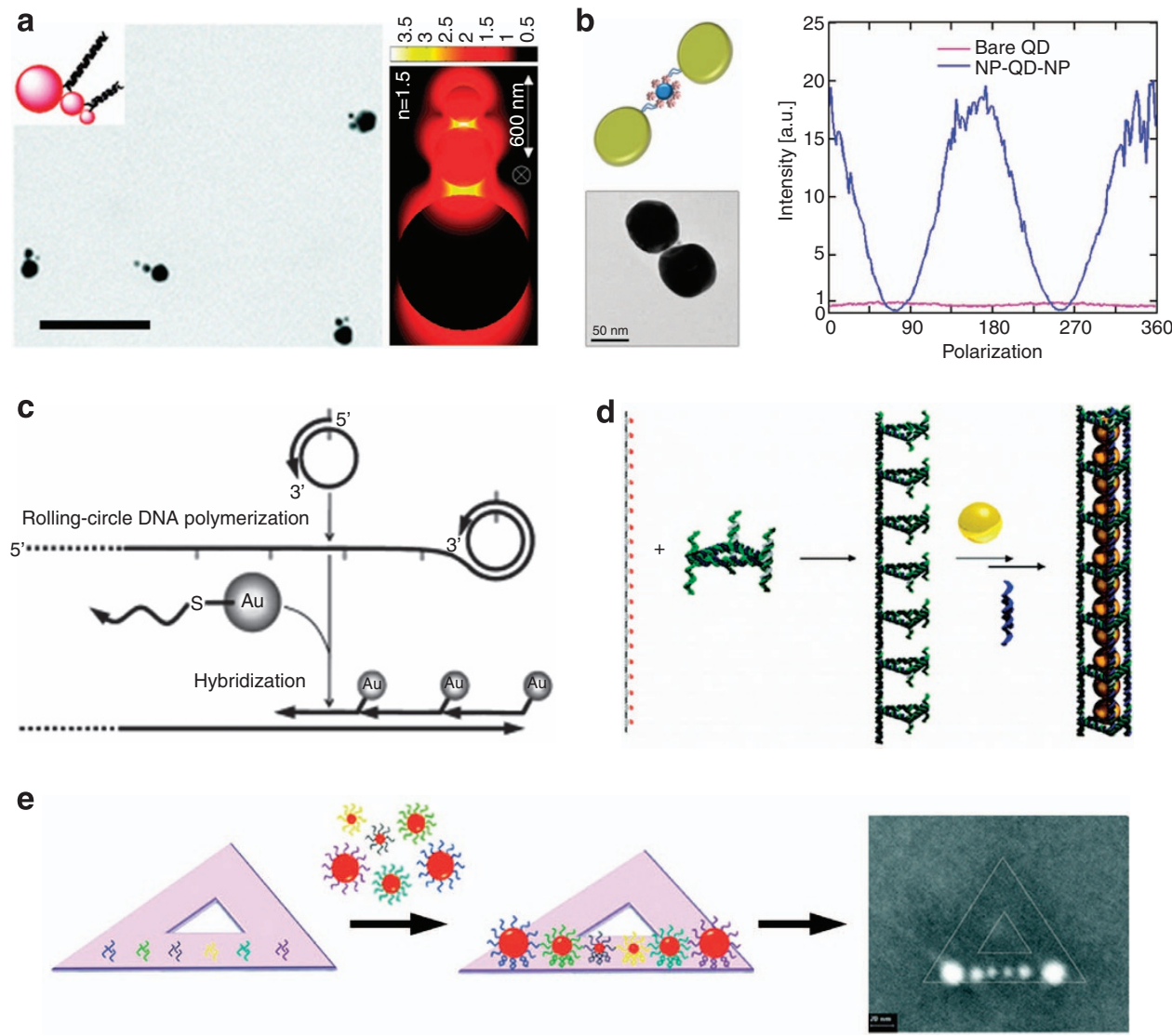

Figure 1 DNA-programmed 1D photonic nanoarchitectures. (a) AuNP trimer nanoarchitecture with interparticle spacing of $<1 \mathrm{~nm}$. The scale bar is $100 \mathrm{~nm}$. Inset shows the EM field distribution. Adapted from reference 28 (2008 ACS). (b) A AuNP dimer with a QD at the nanojunction. The emissionenhancement factor is dependent on polarization of the incident EM field. Adapted from reference 31 (2012 ACS). (c) Rolling-circle DNA polymerization directed the production of AuNP linear aggregates at micrometer scale. Adapted from reference 33 (2005 Wiley-VCH). (d) DNA triangular nanotube templated AuNP chain with predetermined length. Adapted from reference 36 (2010 ACS). (e) AuNP self-similar chain assembled on triangular DNA origami. Adapted from reference 39 (2010 ACS). 
enhancement factor approached the peaked maximum when the incident EM field was parallel to the dimer axis (Figure 1b). This is associated with the strong EM field enhancement, which originates from the occurrence of longitudinal-bonding plasmon mode. In addition, fluorescence molecules in the vicinity of MNPs can also exhibit enhanced emission resulting from near-field plasmon-dipole interactions. Tinnefeld and coworkers ${ }^{32}$ observed enhancement of up to 100 -fold for a single molecule emitter which was docked in the nanogap of 100-nm AuNP dimers bound on a DNA origami pillar.

To investigate the plasmon modes of the linear nanoarchitecture, producing longer assemblies with structural rigidity and defined length is necessary. Rolling-circle DNA polymerization was employed to build linear AuNP nanoarchitectures whose length were up to micrometers, but the structural deformations of the products were inevitable as a result of the flexibility of the DNA duplex template (Figure 1c). ${ }^{33}$ Symmetric surface modification of AuNPs with DNA is an efficient strategy to facilitate the assembly of linear nanoarchitectures, which is driven by base-pair recognition between the DNA ligands on AuNP surface. Linear AuNP arrays have been obtained by using the divalent DNA-AuNP conjugates as building blocks, in which DNA were immobilized at diametrically opposite binding sites on AuNP surface. ${ }^{34,35}$ On the other hand, a robust template with sufficient structural rigidity readily enables organizing AuNPs into linear nanoarchitectures. Sleiman and coworkers ${ }^{36}$ explored a DNA triangular nanotube comprising three duplex DNA to improve the structural rigidity of the template. (Figure 1d). The periodic cavities in the nanotube allows for controlled encapsulation of AuNPs, generating linear AuNP nanoarchitectures with tunable length. The plasmon couplings within such longer linear assembly make it function as a whole when interacting with the incident light. As a result, two plasmon modes were generated, one is a strong longitudinal mode that is parallel to the axis and another is a weak transverse mode that is perpendicular to the axis. The strong longitudinal mode leads to a new peak in the red wavelength region of the plasmon band.

In recent years, DNA origami invented by Rothemund, ${ }^{37}$ has proved to be a powerful template in producing linear photonic nanoarchitectures with high precision and structural rigidity. DNA origami explores a long single stranded DNA as a molecular scaffold that is folded along a predetermined path by hybridizing with a set of short staple strands. DNA origami features high structural rigidness and precise addressability that are significantly advantageous for directed assembly of DNA surface-engineered nanomaterials. The structural parameters of the assembled nanoarchitectures, such as spatial configurations and interparticle distances, can be precisely tailored by rationally designing the functional DNA strands on the origami template. Yan and coworkers ${ }^{38}$ proved the organizational power of DNA origami by building discrete AgNP dimers and trimers on a triangular DNA origami. In this study, AgNPs of $20 \mathrm{~nm}$ were functionalized with single stranded DNA, which has a segment complimentary to the capturing strands on the origami. Through base-pair hybridization between the DNA strands capped on the AgNP surface and the DNA capturing strands on the origami, AgNP dimer and trimer nanoarchitectures were generated as a result. Later, six AuNPs of various sizes (15, 10 and $5 \mathrm{~nm}$ ) were successfully organized into self-similar chain nanoarchitectures on a triangular DNA origami (Figure 1e). ${ }^{39}$ More recently, Klein et al. ${ }^{40}$ constructed AuNP waveguides by using multi-DNA origami scaffold which greatly improves the mechanical rigidity and the linearity of the architectures. Also, QD nanoarchitectures were assembled on DNA origami tube through streptavidin-biotin recognition between streptavidin-capped QDs and the biotin molecules on the origami tube. ${ }^{41}$

\section{TWO DIMENSIONAL DNA-PROGRAMMED PHOTONIC NANOARCHITECTURES}

According to extensive theoretical studies on 2D discrete metal nanoarchitectures, the in-phase charge oscillations that yield a large dipole moment can give rise to a bright super-radiant plasmon mode. Surface charge oscillations oriented in different directions, which result in a small total dipole moment, produce a dark subradiant mode. ${ }^{42,43}$ Thus, controlled organization of MNPs into well-defined 2D discrete nanoarchitectures using DNA-programmed self-assembly provides us opportunities to tune the radiative and nonradiative plasmonic properties. A hexagon DNA template whose geometry is defined by corner organic molecules introduced in DNA strands can be used to build discrete hexagonal AuNP nanoarchitectures. ${ }^{44}$ Attaching a AuNP specifically to the interior or terminal $\left(5^{\prime}\right.$ or $\left.3^{\prime}\right)$ site of 2D DNA geometries, such as triangles and squares, enables constructing a series of $2 \mathrm{D}$ discrete nanoarchitectures. ${ }^{45}$ Capasso and coworkers $^{46}$ assembled a heteropentamer cluster composed of a AuNP surrounded by a ring of four larger AuNPs through DNA hybridization (Figure 2a). In this pentamer, bright mode yields large cluster dipole moments and the mode strongly redshifts owing to strong capacitive coupling between neighboring NPs. Capacitive coupling between the AuNP and the two nanoshells above and below the AuNP redshifts the dark mode close to the bright mode peak. As a result, a narrow Fano dip was generated in the extinction spectra of such pentamer, resulting from the interference between the bright mode and the dark mode that have overlap in frequency.

Despite the 2D discrete nanoarchitectures made of isotropic spherical nanoparticles, Yan and coworkers ${ }^{47}$ reported 2D gold nanorod (AuNR) dimers with varied inter-rod angles on a triangular DNA origami. The end-to-end arrangements of AuNRs generated a strong longitudinal plasmon coupling, which created a red shift of absorption spectra compared with single AuNR. Whereas, the side-by-side arrangement of AuNRs created a blue shift of absorption spectra compared with single AuNR, resulting from the strong transverse plasmon coupling. These 2D architectures made of 1D building blocks with defined geometry and boundary are of fundamental significance for developing the electronic and/or optoelectronic devices. Wang and coworkers ${ }^{48}$ reported a typical example of a T-shaped 2D nanoarchitecture comprising three AuNRs and one AuNP assembled on a rectangular DNA origami, in which the AuNRs and the AuNP act as the source, the drain and the gate electrodes and the Coulomb island, respectively. This T-shaped AuNR nanoarchitecture holds great promise for functioning as the core device in room temperature single-electron transistor (Figure $2 b$ ).

When MNPs are organized into 2D extended nanoarchitectures beyond the discrete geometry discussed above, in particular, 2D periodic arrays, the presence of order will give rise to excitation of collective modes, which can strongly affect the radiative character of the plasmonic resonances of the individual NPs. This allows us to modify the far-field properties by assembling MNPs into periodic arrays. $^{2,49}$ In this respect, a large assortment of periodic 2D AuNP nanoarchitectures have been built based on DNA tile systems, although few systematic investigations on their plasmon resonance property have been reported. For example, Yan and coworkers ${ }^{50}$ rationally designed a two-tile system to assembly $5 \mathrm{~nm}$ AuNPs specifically in one tile to form different 2D AuNP lattice structures. Kiehl and coworkers ${ }^{51}$ reported 2D AuNP nanoarrays that were characterized by high density of NPs and precisely-defined interrow spacing by hybridizing DNA functionalized AuNPs to a preassembled 2D DNA scaffold in situ on a solid surface. Further, they designed four different double-crossover (DX) tiles to assemble into 2D DNA 

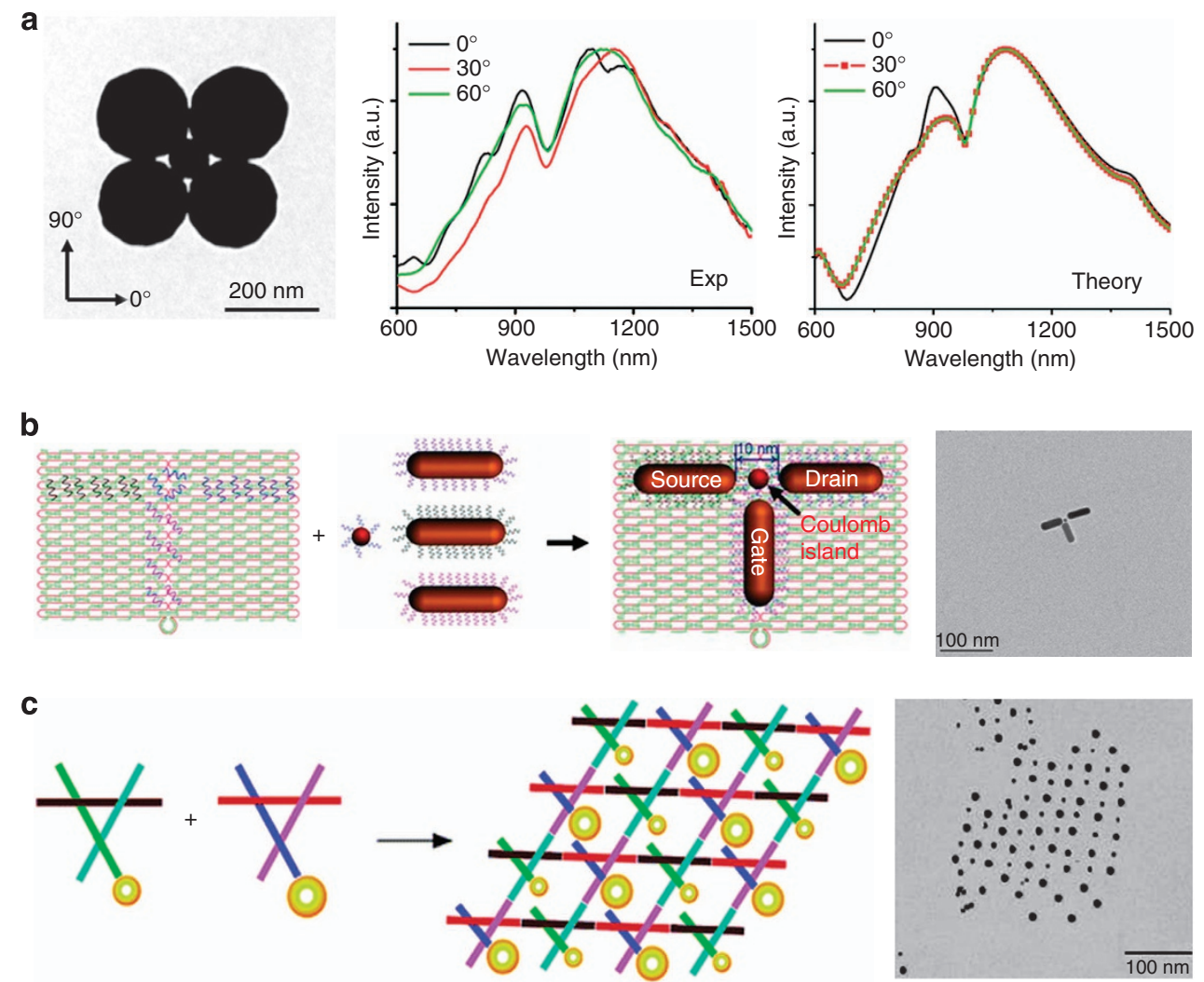

Figure 2 DNA-programmed 2D photonic nanoarchitectures. (a) A DNA-guided metal pentamer. A fano dip is observed in the extinction spectra of the pentamer. Adapted from reference 46 (2011 ACS). (b) DNA origami-directed ' $T$ '-shaped architecture resembling the core structure of a room temperature single-electron transistor. Adapted from reference 48 (2013 Wiley- $\mathrm{VCH}$ ). (c) AuNP nanoarrays assembled from six-arm motif/AuNP conjugates. Adapted from reference 24 (2006 ACS).

scaffolding and realized the precise arrangement of multiple DNAencoded AuNPs into 2D AuNP nanoarrays consisting of alternating rows of different-sized AuNPs. ${ }^{52}$ Later, Seeman and coworkers ${ }^{24}$ designed robust three-space-spanning DNA motifs to organize 5 and $10 \mathrm{~nm}$ AuNPs into 2D periodically ordered nanoarchitectures (Figure 2c).

In addition to the above DNA-programmed 2D discrete and periodic metallic nanoarchitectures and their associated optical properties, DNA-directed 2D discrete QD homo-nanoarchitectures and MNP-QD hetero-nanoarchitectures have also been studied. Upon rationally organizing QDs that are compatible in band-gap energy, we can harvest and guide the light energy flow along propagation path from wide band-gap to narrow band-gap QDs. A clear experimental demonstration of QD-based light guiding was shown by Kelley and coworkers. ${ }^{8}$ In their study, the QDs were functionalized with rationally designed DNA ligands that consist of three domains: a QD-binding domain featuring phosphorothioate linkages within the backbone, a spacer containing phosphodiester linkages, and a DNAbinding domain also composed of a sequence with phosphodiester linkages. The inclusion of 20 guanine units in the QD-binding domain produced QDs with one DNA molecule per QD, while 10, 7 and 5 guanine units produced valencies of 2, 3 and 4, respectively. These QDs of polyvalent forms were subsequently assembled into 2D branched complex, in which wide band-gap QDs were placed at the terminus and narrow band-gap QDs at the center. As a result, the emission energy was found to be directionally transferred from the exterior QDs to the centered QDs. Similarly, branched hetero-nanoarchitectures with QD surrounded by defined number of AuNPs were also reported through DNA ligand-specific recognition. ${ }^{53}$ These architectures have considerable potentials in exploring the plasmon-exciton interactions and manipulation of the optical modes of emission in a quantitative manner. In addition, stable core/shell QD-DNA conjugates with high salt resistance have been synthesized by inserting the end of the phosphorothiolated oligonucleotide (5-10 nucleotides) into the shell of the QD. This certainly opens up a new avenue for DNA-guided precise positioning of QDs, especially based on DNA origami templates which require a highly-salted buffered condition. ${ }^{54}$

\section{THREE DIMENSIONAL DNA-PROGRAMMED PHOTONIC NANOARCHITECTURES}

In $3 \mathrm{D}$ photonic nanoarchitectures, their plasmonic chirality has received tremendous interest, which arises from the fact that, in chiral arrangements of MNPs, the plasmon extinction differs for leftand right-handed circularly polarized light. Considering a 3D spiral MNP nanoarchitecture, the helical arrangement of plasmonic dipoles of MNPs gives rise to increased absorption of the incident circularly polarized light that has the same handedness. The difference in absorption of left- and right-handed circularly polarized light generates circular dichroism (CD) with features in the plasmon resonance wavelength region. In this area, DNA-programmed scaffolds have been widely exploited to build 3D chiral nanoarchitectures with the hope of tailoring the chiral optical effects through modifying the $3 \mathrm{D}$ structural configurations. 
As well known, in many chiral organic molecules, the chiroptical effects are related to the tetrahedral bonding structure of carbon. Similarly, metallic tetramer that cannot be brought to coincide with its mirror-symmetric counterpart is expected to show chiral optical activity. Both pyramidal homo-tetramers made of AuNPs and pyramidal hetero-tetramers made of AuNPs, AgNPs and QDs have been built through specific base paring between DNA monofunctionalized nanoparticles, ${ }^{55,56}$ in which each DNA strand travels through three pyramid sides and traces out one face of the pyramid. Kotov and coworkers ${ }^{56}$ observed apparent chiral spectra reversal from those mirror-symmetric pyramidal nanoarchitectures. Upon introduction of multiple particles in the pyramid, which leads to high degree of the asymmetric geometry, the chiral optical signals were greatly enhanced. Inclusion of AgNP that supports larger surface plasmon resonance also enhanced the resultant chiroptical activity of the pyramid. AuNP tetramers which exhibited chiral optical response at the plasmon resonance wavelength were also realized by precisely positioning AuNPs on a rectangular DNA origami through base-pair recognition between DNA-capping strands on AuNP surface and DNA capturing strands on origami. ${ }^{57}$ To obtain chiral MNP nanoarchitectures consisting of $3 \mathrm{D}$ spirals, Liedl and coworkers ${ }^{58}$ used a 24-DNA helix bundle to direct the positioning of AuNPs, in which helically arranged attachment sites are displayed on the bundle. (Figure 3a). They found that the CD signals of such helical nanoarchitectures can be strengthened by growing silver on the AuNPs, which can be attributed to the greatly increased optical absorption caused by the strong plasmonic dipoles of Au/Ag coreshell NPs. This matches well with the observation in previously mentioned pyramidal hetero-tetramers. Besides, twisting two linear rows of AuNPs that were preorganized on a rectangular DNA origami can also lead to a helical arrangement of AuNPs. ${ }^{59}$

Extensive theoretical and experimental studies pointed out that the chiral optical behavior of photonic nanoarchitectures is significantly affected by the anisotropic factors of the constituent building
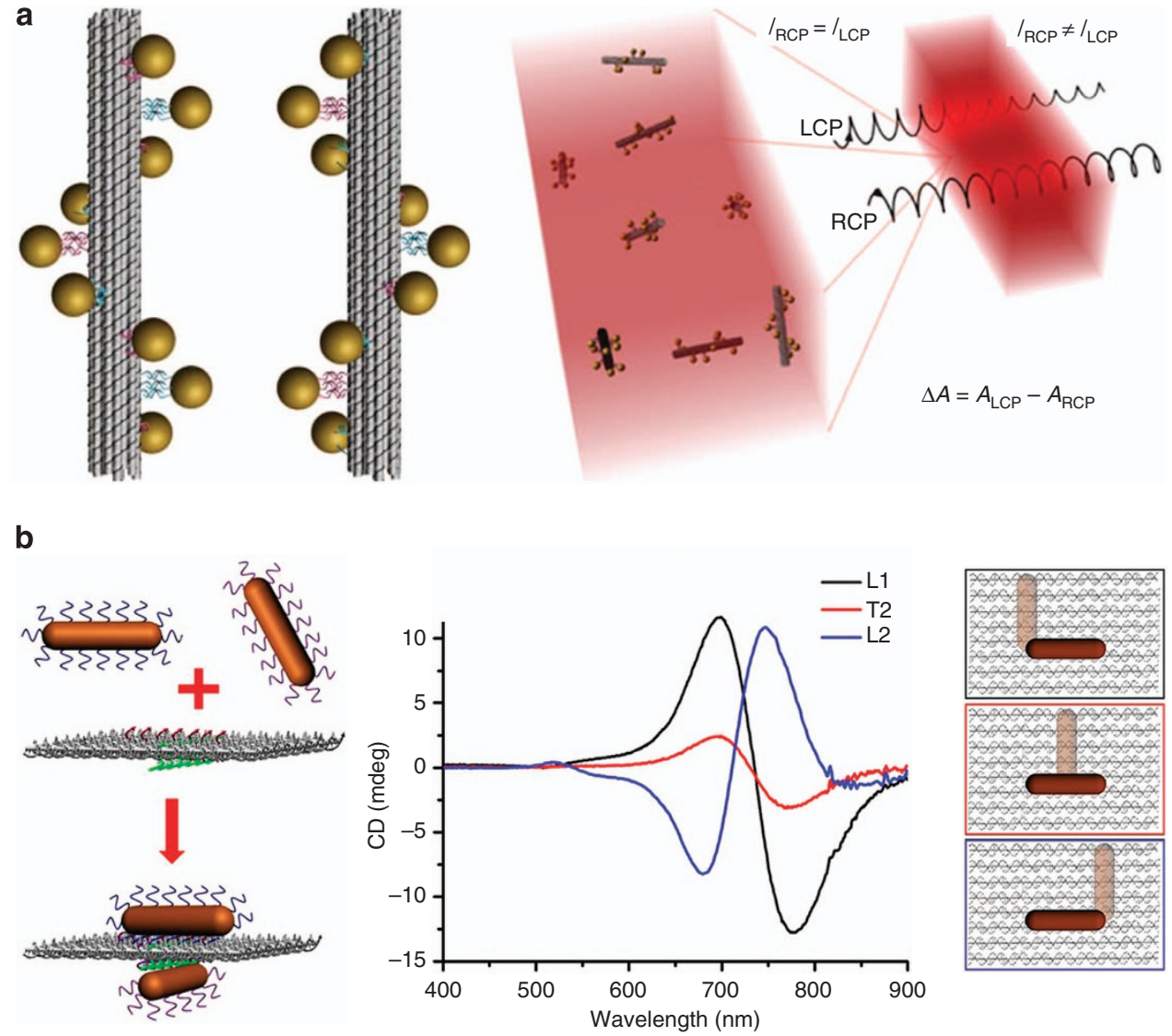

C
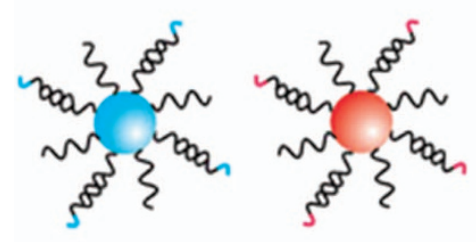

b.c.c.

Figure 3 DNA-programmed 3D photonic nanoarchitectures. (a) AuNP helix assembled on DNA origami tube. The difference in absorption of left- and righthanded circularly polarized light generates circular dichroism. Adapted from reference 58 (2012 NPG). (b) Bifacial DNA origami-directed 3D, discrete AuNR dimer nanoarchitecture with tunable plasmonic chirality. Adapted from reference 63 (2013 ACS). (c) DNA-programmed body-centered cubic (bcc) AuNP superlattice crystals and its SAXS pattern. Adapted from reference 69 (2008 NPG). 
blocks. ${ }^{60,61}$ Amorphous 3D AuNR aggregates, which are prepared through base-pair hybridization between DNA linker strands and DNA-capping strands on NR surface, can generate remarkable plasmonic CD signals at the wavelength region of longitudinal plasmon mode of AuNRs. ${ }^{62}$ More recently, Wang and coworkers ${ }^{63}$ presented a great progress in construction of 3D nanoarchitectures consisting of anisotropic building blocks with tailored plasmonic chirality, where 3D AuNR discrete dimers with defined structural configuration were built using bifacial 2D DNA origami as template (Figure 3b). Strong chiral optical signals were observed from ' $\mathrm{L}$ '-shaped asymmetric 3D dimers. This plasmonic chirality of the 3D AuNR dimer is strongly sensitive to the structural configuration, which makes it a good candidate as chiral plasmonic ruler.

In recent years, 3D metamaterials with operating frequency at optical regime have been greatly progressed for their intriguing photonic applications, such as negative index materials, enhanced nonlinear effects and broadband circular polarizers. ${ }^{64-66}$ Conventional top-down lithography, for examples, stacked electron-beam lithography and direct laser writing, were widely used to make those periodic 3D photonic metamaterials. Whereas, DNAprogrammed self-assembly is another powerful strategy that can lead to complex 3D photonic materials with rationally-tailored compositions and spatial characteristics. A large collection of 3D DNA-guided photonic superlattice crystals have been realized with tunable lattice parameters. ${ }^{67}$ The collective optical properties of these artificial 3D superlattice of crystals, which arise from the near-field interactions among their individual components at $3 \mathrm{D}$ space, are expected to be tuned by choosing different building blocks and designing the route of assembly. ${ }^{68}$ The first step toward DNA-guided 3D ordered superlattice was pioneered by Mirkin ${ }^{69}$ and Gang. ${ }^{70}$ Mirkin and coworkers ${ }^{69}$ used DNA to control the crystallization of AuNP-DNA conjugates to the extent that different DNA sequences guide the assembly of the same type of AuNP into micrometer-sized face-centered-cubic or body-centered-cubic crystal structures (Figure 3c). Gang and coworkers, formed 3D crystalline assemblies of AuNPs mediated by interactions between complementary DNA molecules attached to the AuNP surface and the 3D AuNP crystals could form reversibly during heating and cooling cycles. ${ }^{70}$ Further, Gang and coworkers ${ }^{71}$ successfully assembled binary heterogeneous body-centered-cubic crystal lattice by hybridizing AuNPs and QDs that were modified by multiple copies of DNA. In addition, the DNAprogrammed 3D superlattice crystals can be tuned dynamically through hybridization or dehybridization reaction between a loop sequence in the DNA linker and external strand. ${ }^{72}$

\section{PERSPECTIVE AND OUTLOOK}

A plethora of new possibilities will arise in manipulating the lightmatter interactions when the photonic building blocks are organized into well-defined nanoarchitectures, where DNA-programmed selfassembly technique has proved to be a robust solution. However, surface chemical encoding of photonic building blocks using DNA ligand, such as asymmetric surface decoration of spherical nanoparticles and site-selective functionalization of anisotropic nanoparticles, has not kept up with the pace of progress in chemical fabrication for nanophotonics. These difficulties prevent us from fully capitalizing on the vast library of available photonic building blocks varying in compositions and shapes so as to build more sophisticated photonic nanoarchitectures. With these insights, flexible strategy for DNA encoding of building blocks with site selectivity and stoichiometry should be developed.
DNA-programmed self-assembly technique allows building photonic nanoarchitectures at a much finer length scale than that by conventional lithographical fabrication, which shows great potential for constructing photonic or optoelectronic nanodevices that feature rational design and high precision. To date, integration of DNAprogrammed photonic nanoarchitectures with 'top-down' lithographically fabricated features is still a major impediment in the photonic application of DNA-programmed self-assembly technique. The challenges lie in the structure preservation of DNA-programmed nanoarchitectures and well-controlled combination of these nanoarchitectures with lithographical features. Fortunately, some progress has been made in this aspect, which can be exemplified in placing DNA origami-templated AuNP 2D nanostructures on lithographically patterned substrates. ${ }^{73}$ These extended 2D metallic nanostructures supported by solid surface are of fundamental significance in realization of plasmonic circuits that can be used for future miniaturized optoelectronic devices. ${ }^{74}$

\section{CONFLICT OF INTEREST}

The authors declare no conflict of interest.

\section{ACKNOWLEDGEMENTS}

QW acknowledges funding by Chinese Academy of Sciences Bairen Ji Hua Program, the National Natural Science Foundation of China (Grant No. 21073225, 91023038), the Natural Science Foundation of Jiangsu province (Grant No. BK2012007), the Chinese Ministry of Science and Technology (2011CB965004), and the CAS/SAFEA International Partnership Program for Creative Research Teams.

1 Kim, S.-H., Lee, S. Y., Yang, S.-M. \& Yi, G.-R. Self-assembled colloidal structures for photonics. NPG Asia Mater. 3, 25-33 (2011).

2 Giannini, V., Fernandez-Domínguez, A. I., Heck, S. C. \& Maier, S. A. Plasmonic nanoantennas: fundamentals and their use in controlling the radiative properties of nanoemitters. Chem. Rev. 111, 3888-3912 (2011).

3 Schuller, J. A., Barnard, E. S., Cai, W., Jun, Y. C., White, J. S. \& Brongersma, M. L. Plasmonics for extreme light concentration and manipulation. Nat. Mater. 9, 193-204 (2010).

4 Gramotnev, D. K. \& Bozhevolnyi, S. I. Plasmonics beyond the diffraction limit. Nat. Photonics 4, 83-91 (2010).

5 Zhang, J., Tang, Y., Lee, K. \& Ouyang, M. Tailoring light-matter-spin interactions in colloidal hetero-nanostructures. Nature 466, 91-95 (2010).

6 Gao, B., Arya, G. \& Tao, A. R. Self-orienting nanocubes for the assembly of plasmonic nanojunctions. Nat. Nanotech. 7, 433-437 (2012).

7 Taubert, R., Hentschel, M., Kästel, J. \& Giessen, H. Classical analog of electromagnetically induced absorption in plasmonics. Nano Lett. 12, 1367-1371 (2012).

8 Tikhomirov, G., Hoogland, S., Lee, P. E., Fischer, A., Sargent, E. H. \& Kelley, S. O. DNA-based programming of quantum dot valency, self-assembly and luminescence. Nat. Nanotech. 6, 485-490 (2011).

9 El-Sayed, M. A. Some interesting properties of metals confined in time and nanometer space of different shapes. Acc. Chem. Res. 34, 257-264 (2001).

10 Jain, P. K., Huang, W. \& El-Sayed, M. A. On the universal scaling behavior of the distance decay of plasmon coupling in metal nanoparticle pairs: a plasmon ruler equation. Nano Lett. 7, 2080-2088 (2007).

11 Prodan, E., Radloff, C., Halas, N. J. \& Nordlander, P. A hybridization model for the plasmon response of complex nanostructures. Science 302, 419-422 (2003).

12 Wang, Y. \& Herron, N. Nanometer-sized semiconductor clusters: materials synthesis, quantum size effects, and photophysical properties. J. Phys. Chem. 95, 525-532 (1991).

13 Peng, X., Schlamp, M. C., Kadavanich, A. V. \& Alivisatos, A. P. Epitaxial growth of highly luminescent $\mathrm{CdSe} / \mathrm{CdS}$ core/shell nanocrystals with photostability and electronic accessibility. J. Am. Chem. Soc. 119, 7019-7029 (1997).

14 Cortie, M. B. \& McDonagh, A. M. Synthesis and optical properties of hybrid and alloy plasmonic nanoparticles. Chem. Rev. 111, 3713-3735 (2011).

15 Nikoobakht, B. \& El-Sayed, M. A. Preparation and growth mechanism of gold nanorods (NRs) using seed-mediated growth method. Chem. Mater. 15, 1957-1962 (2003).

16 Brinson, B. E., Lassiter, J. B., Levin, C. S., Bardhan, R., Mirin, N. \& Halas, N. J. Nanoshells made easy: improving Au layer growth on nanoparticle surfaces. Langmuir 24, 14166-14171 (2008).

17 Jana, N. R., Gearheart, L. \& Murphy, C. J. Seeding growth for size control of 5-40 nm diameter gold nanoparticles. Langmuir 17, 6782-6786 (2001). 
18 Rycenga, M., Cobley, C. M., Zeng, J., Li, W., Moran, C. H., Zhang, Q., Qin, D. \& Xia, Y. Controlling the synthesis and assembly of silver nanostructures for plasmonic applications. Chem. Rev. 111, 3669-3712 (2011).

19 Shen, S., Zhang, Y., Peng, L., Du, Y. \& Wang, Q. Matchstick-shaped Ag2 S-ZnS heteronanostructures preserving both UV/blue and near-infrared photoluminescence. Angew. Chem. Int. Ed. 50, 7115-7118 (2011).

20 Du, Y., Xu, B., Fu, T., Cai, M., Li, F., Zhang, Y. \& Wang, Q. Near-infrared photoluminescent $\mathrm{Ag}_{2} \mathrm{~S}$ quantum dots from a single source precursor. J. Am. Chem. Soc. 132, 1470-1471 (2010).

21 Benson, 0 . Assembly of hybrid photonic architectures from nanophotonic constituents. Nature 480, 193-199 (2011)

$22 \mathrm{Nie}, \mathrm{Z}$., Petukhova, A. \& Kumacheva, E. Properties and emerging applications of selfassembled structures made from inorganic nanoparticles. Nat. Nanotech. 5, 15-25 (2010).

23 Lan, X., Chen, Z., Liu, B., Ren, B., Henzie, J. \& Wang, Q. DNA-directed gold nanodimers with tunable sizes and interparticle distances and their surface plasmonic properties. Small 9, 2308-2315 (2013).

24 Zheng, J., Constantinou, P. E., Micheel, C., Alivisatos, A. P., Kiehl, R. A. \& Seeman, N. C. Two-dimensional nanoparticle arrays show the organizational power of robust DNA motifs. Nano Lett. 6, 1502-1504 (2006).

25 Sharma, J., Chhabra, R., Cheng, A., Brownell, J., Liu, Y. \& Yan, H. Control of selfassembly of DNA tubules through integration of gold nanoparticles. Science 2, 112-116 (2009).

26 Alivisatos, A. P., Johnsson, K. P., Peng, X., Wilson, T. E., Loweth, C. J., Bruchez, M. P. \& Schultz, P. G. Organization of 'nanocrystal molecules' using DNA. Nature 382, 609-611 (1996).

27 Sheikholeslami, S., Jun, Y., Jain, P. K. \& Alivisatos, A. P. Coupling of optical resonances in a compositionally asymmetric plasmonic nanoparticle dimer. Nano Lett. 10, 2655-2660 (2010).

28 Bidault, S., García de Abajo, F. J. \& Polman, A. Plasmon-based nanolenses assembled on a well-defined DNA template. J. Am. Chem. Soc. 130, 2750-2751 (2008).

29 Reinhard, B. M., Siu, M., Agarwal, H., Alivisatos, A. P. \& Liphardt, J. Calibration of dynamic molecular rulers based on plasmon coupling between gold nanoparticles. Nano Lett. 5, 2246-2252 (2005).

30 Muskens, O. L., Giannini, V., Sánchez-Gil, J. A. \& Gómez Rivas, J. Strong enhancement of the radiative decay rate of emitters by single plasmonic nanoantennas. Nano Lett. 7 , 2871-2875 (2007)

31 Cohen-Hoshen, E., Bryant, G. W., Pinkas, I., Sperling, J. \& Bar-Joseph, I. Excitonplasmon interactions in quantum dot-gold nanoparticle structures. Nano Lett. 12, 4260-4264 (2012).

32 Acuna, G. P. Möller, F. M., Holzmeister, P., Beater, S., Lalkens, B. \& Tinnefeld, P. Fluorescence enhancement at docking sites of DNA-directed self-assembled nanoantennas. Science 338, 506-510 (2012).

33 Deng, Z., Tian, Y., Lee, S.-H., Ribbe, A. E. \& Mao, C. DNA-encoded self-assembly of gold nanoparticles into one-dimensional arrays. Angew. Chem. Int. Ed. 117, 3648-3651 (2005)

34 Ohya, Y., Miyoshi, N., Hashizume, M., Tamaki, T., Uehara, T., Shingubara, S. \& Kuzuya, A. Formation of $1 \mathrm{D}$ and 2D gold nanoparticle arrays by divalent DNA-gold nanoparticle conjugates. Small 8, 2335-2340 (2012).

35 Zhang, T., Dong, Y., Sun, Y., Chen, P., Yang, Y., Zhou, C., Xu, L., Yang, Z. \& Liu, D. DNA bimodified gold nanoparticles. Langmuir 28, 1966-1970 (2012).

36 Lo, P. K., Altvater, F. \& Sleiman, H. F. Templated synthesis of DNA nanotubes with controlled predetermined lengths. J. Am. Chem. Soc. 132, 10212-10214 (2010).

37 Rothemund, P. W. K. Folding DNA to create nanoscale shapes and patterns. Nature 440, 297-302 (2006)

38 Pal, S., Deng, Z., Ding, B., Yan, H. \& Liu, Y. DNA-origami-directed self-assembly of discrete silver-nanoparticle architectures. Angew. Chem. Int. Ed. 49, 2700-2704 (2010).

39 Ding, B., Deng, Z., Yan, H., Cabrini, S. Zuckermann, R. N. \& Bokor, J. Gold nanoparticle self-similar chain structure organized by DNA origami. J. Am. Chem. Soc. 132, 3248-3249 (2010).

40 Klein, W. P., Schmidt, C. N., Rapp, B., Takabayashi, S., Knowlton, W. B., Lee, J., Yurke, B., Hughes, W. L., Graugnard, E. \& Kuang, W. Multiscaffold DNA origami nanoparticle waveguides. Nano Lett. 13, 3850-3856 (2013).

41 Bui, H., Onodera, C., Kidwell, C., Tan, Y., Graugnard, E., Kuang, W., Lee, J., Knowlton, W. B., Yurke, B. \& Hughes, W. L. Programmable periodicity of quantum dot arrays with DNA origami nanotubes. Nano Lett. 10, 3367-3372 (2010).

42 Lovera, A., Gallinet, B., Nordlander, P. \& Martin, O. J. F. Mechanisms of Fano resonances in coupled plasmonic systems. ACS Nano 7, 4527-4536 (2013).

43 Verellen, N., Sonnefraud, Y., Sobhani, H., Hao, F., Moshchalkov, V. V., Dorpe, P. V. \& Nordlander, P. Fano resonances in individual coherent plasmonic nanocavities. Nano Lett. 9, 1663-1667 (2009).

44 Aldaye, F. A. \& Sleiman, H. F. Sequential self-assembly of a DNA hexagon as a template for the organization of gold nanoparticles. Angew. Chem. Int. Ed. 118, 2262-2267 (2006).

45 Wen, Y., McLaughlin, C. K., Lo, P. K., Yang, H. \& Sleiman, H. F. Stable gold nanoparticle conjugation to internal DNA positions: facile generation of discrete gold nanoparticle-DNA assemblies. Bioconjugate Chem. 21, 1413-1416 (2010).

46 Fan, J. A., He, Y., Bao, K., Wu, C., Bao, J., Schade, N. B., Manoharan, V. N., Shvets, G., Nordlander, P., Liu, D. R. \& Capasso, F. DNA-enabled self-assembly of plasmonic nanoclusters. Nano Lett. 11, 4859-4864 (2011).

47 Pal, S., Deng, Z., Wang, H., Zou, S., Liu, Y. \& Yan, H. DNA directed self-assembly of anisotropic plasmonic nanostructures. J. Am. Chem. Soc. 133, 17606-17609 (2011).
48 Chen, Z., Lan, X. \& Wang, Q. DNA origami directed large-scale fabrication of nanostructures resembling room temperature single-electron transistors. Small 9, 3567-3571 (2013).

49 Auguié, B. \& Barnes, W. L. Collective resonances in gold nanoparticle arrays. Phys. Rev. Lett. 101, 143902 (2008)

50 Sharma, J., Chhabra, R., Liu, Y., Ke, Y. \& Yan, H. DNA-templated self-assembly of two-dimensional and periodical gold nanoparticle arrays. Angew. Chem. Int. Ed. 45, 730-735 (2006)

51 Le, J. D., Pinto, Y., Seeman, N. C., Musier-Forsyth, K., Taton, T. A. \& Kiehl, R. A. DNAtemplated self-assembly of metallic nanocomponent arrays on a surface. Nano Lett. 4, 2343-2347 (2004).

52 Pinto, Y. Y., Le, J. D., Seeman, N. C., Musier-Forsyth, K., Taton, T. A. \& Kiehl, R. A. Sequence-encoded self-assembly of multiple-nanocomponent arrays by 2D DNA scaffolding. Nano Lett. 5, 2399-2402 (2005).

$53 \mathrm{Fu}$, A. H., Micheel, C. M., Cha, J., Chang, H., Yang, H. \& Alivisatos, A. P. Discrete nanostructures of quantum dots/Au with DNA. J. Am. Chem. Soc. 126, 10832-10833 (2004).

54 Deng, Z., Samanta, A., Nangreave, J., Yan, H. \& Liu, Y. Robust DNA-functionalized core/shell quantum dots with fluorescent emission spanning from UV-Vis to near-IR and compatible with DNA-directed self-assembly. J. Am. Chem. Soc 134, 17424-17427 (2012).

55 Mastroianni, A. J., Claridge, S. A. \& Alivisatos, A. P. Pyramidal and chiral groupings of gold nanocrystals assembled using DNA scaffolds. J. Am. Chem. Soc. 131, 8455-8459 (2009).

56 Yan, W., Xu, L., Xu, C., Ma, W., Kuang, H., Wang, L. \& Kotov, N. A. Self-assembly of chiral nanoparticle pyramids with strong R/S optical activity. J. Am. Chem. Soc. 134, 15114-15121 (2012)

57 Shen, X., Asenjo-Garcia, A., Liu, Q., Jiang, Q., Javier García de Abajo, F., Liu, N. \& Ding, B. Three-dimensional plasmonic chiral tetramers assembled by DNA origami. Nano Lett. 13, 2128-2133 (2013).

58 Kuzyk, A., Schreiber, R., Fan, Z., Pardatscher, G., Roller, E.-M., Hoegele, A. Simmel, F. C., Govorov, A. O. \& Liedl, T. DNA-based self-assembly of chiral plasmonic nanostructures with tailored optical response. Nature 483, 311-314 (2012).

59 Shen, X., Song, C., Wang, J., Shi, D., Wang, Z., Liu, N. \& Ding, B. Rolling up gold nanoparticle-dressed DNA origami into three-dimensional plasmonic chiral nanostructures. J. Am. Chem. Soc. 134, 146-149 (2012).

60 Fan, Z. \& Govorov, A. O. Plasmonic circular dichroism of chiral metal nanoparticle assemblies. Nano Lett. 10, 2580-2587 (2010).

61 Guerrero-Martínez, A., Auguié, B., Alonso-Gómez, J. L., Džolić, Z., Gómez-Graña, S., Žinić, M., Cid, M. M. \& Liz-Marzán, L. M. Intense optical activity from threedimensional chiral ordering of plasmonic nanoantennas. Angew. Chem. Int. Ed. 50, 5499-5503 (2011).

62 Li, Z., Zhu, Z., Liu, W., Zhou, Y., Han, B., Gao, Y. \& Tang, Z. Reversible plasmonic circular dichroism of Au nanorod and DNA assemblies. J. Am. Chem. Soc. 134, 3322-3325 (2012).

63 Lan, X., Chen, Z., Dai, G., Lu, X., Ni, W. \& Wang, Q. Bifacial DNA origami-directed discrete, three-dimensional, anisotropic plasmonic nanoarchitectures with tailored optical chirality. J. Am. Chem. Soc. 135, 11441-11444 (2013).

64 Soukoulis, C. M. \& Wegener, M. Past achievements and future challenges in the development of three-dimensional photonic metamaterials. Nat. Photonics 5, 523-530 (2011).

65 McPhedran, R. C., Shadrivov, I. V., Kuhlmey, B. T. \& Kivshar, Y. S. Metamaterials and metaoptics. NPG Asia Mater. 3, 100-108 (2011)

66 Zhao, Y., Belkin, M. A. \& Alù, A. Twisted optical metamaterials for planarized ultrathin broadband circular polarizers. Nat. Commun. 3, 870 (2012).

67 Macfarlane, R. J., Lee, B., Jones, M. R., Harris, N., Schatz, G. C. \& Mirkin, C. A. Nanoparticle superlattice engineering with DNA. Science 334, 204-208 (2011).

68 Jones, M. R., Macfarlane, R. J., Lee, B., Zhang, J., Young, K. L., Senesi, A. J. \& Mirkin, C. A. DNA-nanoparticle superlattices formed from anisotropic building blocks. Nat. Mater. 9, 913-917 (2010)

69 Park, S. Y., Lytton-Jean, A. K. R., Lee, B., Weigand, S., Schatz, G. C. \& Mirkin, C. A. DNA-programmable nanoparticle crystallization. Nature 451, 553-556 (2008).

70 Nykypanchuk, D., Maye, M. M., Lelie, D. \& Gang, O. DNA-guided crystallization of colloidal nanoparticles. Nature 451, 549-552 (2008).

71 Sun, D. \& Gang, O. Binary heterogeneous superlattices assembled from quantum dots and gold nanoparticles with DNA. J. Am. Chem. Soc. 133, 5252-5254 (2011).

72 Maye, M. M., Kumara, M. T., Nykypanchuk, D., Sherman, W. B. \& Gang, O. Switching binary states of nanoparticle superlattices and dimer clusters by DNA strands. Nat. Nanotech. 5, 116-120 (2010).

73 Hung, A. M., Micheel, C. M., Bozano, L. D., Osterbur, L. W., Wallraff, G. M. \& Cha, J. N. Large-area spatially ordered arrays of gold nanoparticles directed by lithographically confined DNA origami. Nat. Nanotech. 5, 121-126 (2010).

74 Ozbay, E. Plasmonics: merging photonics and electronics at nanoscale dimensions. Science 311, 189-193 (2006).

(c) (i) (2) $\ominus$ This work is licensed under a Creative Commons
Attribution-NonCommercial-NoDerivs 3.0 Unported License. To view a copy of this license, visit http://creativecommons. org/licenses/by-nc-nd/3.0/ 


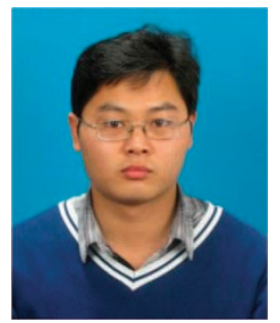

Xiang Lan received his BS from Dong Hua University in 2009. Since then, he joined Professor Qiangbin Wang's group in Suzhou Institute of Nano-Tech and Nano-Bionics, Chinese Academy of Sciences as a $\mathrm{PhD}$ candidate. His present research interest focuses on the DNA-programmed construction of photonic nanostructures.

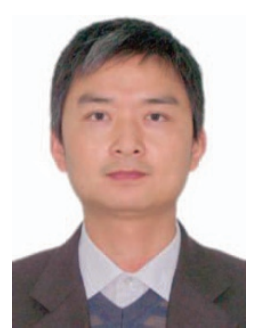

Professor Qiangbin Wang obtained his PhD from East China University of Science and Technology in 2002. Then, he worked as a research associate, postdoctral and assistant research professor at Shanghai Jiaotong University and Arizona State University, respectively. In 2008, he joined Suzhou Institute of Nano-Tech and Nano-Bionics, Chinese Academy of Sciences as a Principal Investigator. His current research interests focus on rational design and modulation of novel optical properties and bioapplications thereof. 